\title{
AMPK isoform expression in the normal and failing hearts
}

\author{
Maengjo Kim ${ }^{1}$, Mei Shen ${ }^{2}$, Soeun Ngoy ${ }^{2}$, Georgios Karamanlidis ${ }^{1}$, Ronglih Liao ${ }^{2}$, and Rong \\ Tian ${ }^{1}$ \\ ${ }^{1}$ Mitochondria and Metabolism Center, Department of Anesthesiology and Pain Medicine, \\ University of Washington, Seattle WA \\ ${ }^{2}$ Cardiovascular division, Department of Medicine, Brigham and Women's Hospital and Harvard \\ Medical School, Boston MA
}

\begin{abstract}
AMP-activated protein kinase (AMPK) is a master metabolic switch that plays an important role in energy homeostasis at the cellular and whole body level, hence a promising drug target. AMPK is a heterotrimeric complex composed of catalytic $\alpha$-subunit and regulatory $\beta$ - and $\gamma$ subunits with multiple isoforms for each subunit. It has been shown that AMPK activity is increased in cardiac hypertrophy and failure but it is unknown whether changes in subunit composition of AMPK contribute to the altered AMPK activity. In this study, we determined the protein expression pattern of AMPK subunit isoforms during cardiac development as well as during cardiac hypertrophy and heart failure in mouse heart. We also compared the findings in failing mouse heart to that of the human failing hearts in order to determine whether the mouse heart is a good model of AMPK in human diseases. In mouse developmental hearts, AMPK was highly expressed in the fetal stages and fell back to the adult level after birth. In the failing mouse heart, there was a significant increase in $\alpha 2, \beta 2$, and $\gamma 2$ subunits both at the mRNA and protein levels. In contrary, we found significant increases in the protein level of $\alpha 1, \beta 1$ and $\gamma 2 \mathrm{c}$ subunits in human failing hearts with no change in the mRNA level. We also compared isoform-specific AMPK activity in the mouse and human failing hearts. Consistent with the literature, in the failing mouse heart, the $\alpha 2$ complexes accounted for $\sim 2 / 3$ of total AMPK activity while the $\alpha 1$ complexes accounted for the remaining 30-35\%. In the human hearts, however, the contribution of a1-AMPK activity were significantly higher $(>40 \%)$ in the non-failing hearts, and it further increased to $50 \%$ in the failing hearts. Thus, the human hearts have a greater amount of $\alpha 1$-AMPK activity compared to the rodent hearts. In summary, the protein level and the isoform distribution of AMPK in the heart change significantly during normal development as well as in heart failure. These observations provide a basis for future development of therapeutic strategies for targeting AMPK.
\end{abstract}

\section{Keywords}

AMPK; human; mice; isoform expression; heart failure

(C) 2012 Elsevier Ltd. All rights reserved.

Correspondence: Rong Tian, MD, PhD, Mitochondria and Metabolism Center, University of Washington School of Medicine, 815 Mercer St., Seattle, WA 98109, Phone: 206-543-8982, Fax: 206-616-4819, rongtian@u.washington.edu.

Disclosures

None.

Publisher's Disclaimer: This is a PDF file of an unedited manuscript that has been accepted for publication. As a service to our customers we are providing this early version of the manuscript. The manuscript will undergo copyediting, typesetting, and review of the resulting proof before it is published in its final citable form. Please note that during the production process errors may be discovered which could affect the content, and all legal disclaimers that apply to the journal pertain. 


\section{Introduction}

AMP-activated protein kinase (AMPK) is a cellular energy sensor and a metabolic master switch that plays a pivotal role in the cell growth and survival as well as in the whole body energy homeostasis [1] [2] [3] . AMPK is activated in response to stresses that deplete intracellular ATP levels leading to a rise in the AMP/ATP ratio. Activation of AMPK depends on the phosphorylation of the Threonine-172 (Thr172) on the catalytic subunit by the upstream kinase LKB-1 or CaMKK $\beta$. Increased AMPK activity leads to phosphorylation of an array of protein targets with the end results of enhanced ATP production and/or reduced energy expenditure [2] [4] [5] [6]. Thus, the AMPK cascade sustains life by constantly adjusting the biological processes of the organism in response to the environmental changes. It has been shown that AMPK pathway is essential for the stress response of the heart. AMPK activity is significantly increased during cardiac stress such as ischemia and pathological cardiac hypertrophy [7] [8], and failure to activate AMPK under these conditions is associated with poor outcome in the heart of mouse models [8] [9] [10] [11] [12].

AMPK is a heterotrimeric complex composed of a catalytic $\alpha$-subunit and two regulatory $\beta$ and $\gamma$-subunits. The $\gamma$-AMPK binds the nucleotides hence functions as the sensor of the kinase. Each subunit exists in multiple isoforms encoded by separate genes $(\alpha 1, \alpha 2, \beta 1, \beta 2$, $\gamma 1, \gamma 2$, and $\gamma 3$ ), and they combine to give rise to a large variety of AMPK holoenzymes. Differences in the expression level and tissue distribution of seven subunit isoforms have been reported but the precise function(s) of these isoforms remain unknown [13] [14] [15]. In the heart, all isoforms of the subunits except $\gamma 3$ are expressed. Based on rodent studies, the $\alpha 2-, \beta 2$ - and $\gamma 1$-subunit are the predominant isoforms in the normal adult heart [14] [15]. There has been no report on whether the isoform profile in the heart changes during physiological or pathological stress conditions. Furthermore, studies of AMPK regulation have so far focused on the phosphorylation of Thr172, and it has not been addressed whether the changes of isoform composition of the heterotrimer constitute a mechanism of regulating the kinase function in the heart.

In this study, we determined the protein expression pattern of AMPK subunit isoforms during cardiac development as well as during cardiac hypertrophy and heart failure in mouse heart. We also compared the finding in mouse failing heart to that of the human failing hearts in order to determine whether the mouse heart is a good model of AMPK in human diseases. We found that there were significant changes in the AMPK expression and isoform distribution pattern in the failing hearts, which resembled the fetal profile. Moreover, the relative contribution of isoform specific AMPK activity in the mouse failing heart was different from that in the human failing heart.

\section{Experimental Procedures}

\section{Animal models}

The mouse heart samples during development were obtained by timed mating. Two female FVB mice were mated with one male FVB mouse in a cage for one night, and the two females were moved to a separate cage and housed until the preset time point of tissue harvest. Mouse heart failure was induced by ascending aortic constriction (AAC) as previously described [16]. At 9 weeks after surgery, the in vivo cardiac function was assessed by echocardiography using a Vevo770 high resolution imaging system with 30MHz RMV-707B scanning head (VisualSonics Inc.). AAC mice demonstrated severe left ventricular dilation and impaired fractional shortening were included in the heart failure group and sham operated mice were used for controls (Table 1). The heart was harvested one day after the echocardiography and was snap-frozen for biochemical assays. 
All animal procedures were performed in accordance with the National Institute of Health's Guide for the Care and Use of Laboratory Animals. The protocols were approved by the institutional IACUC committees at the Brigham and Women's Hospital and the University of Washington.

\section{Human samples}

Myocardial samples were obtained from discarded tissue as previously described [17]. In brief, the failing heart samples (HF) were obtained from the left ventricular (LV) free wall or apex of the heart during implantation of a LV assist device. The nonfailing heart samples (NF) were obtained from the LV free wall of donor hearts found unsuitable for transplantation for a variety of reasons e.g. history of blood transfusion in the emergency room, unacceptable age, or history of resuscitation before death etc. All donors had no history, macroscopic, or laboratory signs of cardiac diseases. The characteristics of the patient population are shown in Table 2 . The study protocol was approved by the IRB committee at the Brigham and Women's Hospital.

\section{Preparation of protein extracts and Immunobloting Analysis}

Protein samples were prepared from frozen heart samples using a lysis buffer containing protease inhibitors (Sigma). Tissue lysates were matched for protein concentration and then separated by SDS-PAGE and transferred onto a polyvinlylidene difluoride membrane (BioRad laboratories). The membranes were blocked in 5\% nonfat milk and incubated with primary antibodies overnight at $4{ }^{\circ} \mathrm{C}: \alpha 1, \alpha 2, \beta 1$ (Epitomics), $\beta 2$ (Cell signal), $\gamma 1$ (Epitomics), and $\gamma 2$ (MBL international). The membranes were incubated with appropriate secondary antibodies conjugated to horseradish peroxidase (HRP) (Pierce) and signal intensities were visualized by Chemiluminescence (Cell signal). Films from at least four independent experiments were scanned and densities of the immunoreactive bands were evaluated using NIH Image software.

\section{RNA isolation and RT-PCR}

Real Time PCR was performed as previously described [17]. Total RNA was isolated from frozen hearts using the RNeasy kit (Qiagen), and cDNA was synthesized using Omniscript reverse synthase and random hexamers according to manufactures guidelines. Real time RCR was performed using SYBR green (Bio-Rad). The primers are described in Table 3. The Real Time PCR results for the mRNA levels of each gene were normalized to $18 \mathrm{~S}$ rRNA levels.

\section{AMPK activity assay and LKB1 treatment}

The isoform specific AMPK activity assay was performed as previously described [12]. The snap-frozen hearts were homogenized, and 100 ug of the lysates were immunoprecipitated with antibodies that recognize specifically $\alpha 1$ or $\alpha 2$ AMPK subunits [12]. For the dephosphorylation experiment, the immunoprecipates were incubated at $37^{\circ} \mathrm{C}$ for 30 minutes in the buffer containing lambda phosphatase (Millipore). For the rephosphorylation experiments, the deposphorylated immnoprecipitates were incubated at $30^{\circ} \mathrm{C}$ for 1 hour in the kinase buffer containing LKB1/STRADa/MO25a complex (Millipore). The kinase reaction was performed in the presence of radioactively labeled ATP with synthetic SAMS peptide as the substrate. AMPK activity is expressed as incorporated ATP (picomoles) per mg protein per minute.

\section{Statistics}

Data were expressed as a mean \pm SD. Comparison between 2 groups was made with 2-tailed Student's $t$ test with unequal variance. $\mathrm{P}<0.05$ was considered statistically significant. 


\section{Results}

\section{AMPK expression and isoform profile during cardiac development in mice}

We examined the protein expression profile of AMPK isoforms during the heart development in mice, from mid-late gestation through the adulthood (Figure 1). All isoforms of the AMPK present in the adult mouse heart were also highly expressed in the fetal heart. The expressions of all subunits except $\beta 1$ were significantly higher in the fetal hearts and the expression fell significantly at day 1 after birth. Notably, the expressions of the $\alpha 1$ catalytic subunit and the $\gamma 2$ nucleotide-binding subunit at E15 were 3-fold higher than adult heart, suggesting that the isoforms of these subunits have switched during the development.

\section{The isoform expression of the AMPK subunits in the failing hearts: mouse vs. man}

We next examined the isoform profiles of the AMPK subunits in the failing hearts of mice and patients. There are two goals of this experiment, first, to determine whether the fetal expression pattern reappears in the failing hearts; and second, to determine whether the AMPK isoform profile in the mouse heart models that of the humans. In the mouse failing heart, there was a significant increase in the mRNA level of $\alpha 2$ (1.5 fold), $\beta 2$ ( 3.5 fold), and $\gamma 2$ (1.9 fold) subunits (Figure 2A). Consistently, the protein level of $\alpha 2$ (2.2 fold), $\beta 2$ (2 fold), and $\gamma 2$ ( 2.5 fold) subunits was increased significantly while the $\beta 1$ isoform decreased by 2 fold (Figure 2B). Similar results were observed in FVB and B57BL6 mice except that the $\gamma 2$ antibody did not recognize the protein in the B57BL6 heart despite a robust mRNA level (Suppl Figure 1).

In contrast to the mouse model, there was no change in the mRNA expression of any AMPK subunit in the human failing heart (Figure 3A) due to idiopathic dilated cardiomyopathy (IDCM) or ischemic cardiomyopathy (ISCM). However, we found significant increases in the protein level of $\alpha 1$ (2- and 1.5-fold for IDCM and ISCM respectively), $\beta 1$ (2- and 1.6fold for IDCM and ISCM respectively) and $\gamma 2 \mathrm{c}$ (4.3- and 3.5-fold for IDCM and ISCM respectively) subunits in human failing hearts (Figure 3B). Three splice variants of $\gamma 2$ subunit have been identified in the human heart (Figure 3B) [18]. Using antibodies specific for the $\mathrm{N}$-terminus of the $\gamma 2$ subunit, we were able to distinguish $\gamma 2 \mathrm{a}$ and $\gamma 2 \mathrm{c}$ variants (Figure 3B). However, the antibody recognizing the c-terminus of the $\gamma$ subunit does not distinguish the $\gamma 1$ from the short form of $\gamma 2$ isoforms $(\gamma 2 b)$. Therefore, we were unable to determine the contribution of $\gamma 2 \mathrm{~b}$ expression to the $\gamma 1$ band in the western blot.

Taken together, in both mouse and human, we found that the overall AMPK protein was increased in the failing heart with significant upregulation of the $\gamma 2$ isoform, as seen in the fetal mouse heart. However the changes of the $\alpha$ and $\beta$ subunits were distinct in human vs. mouse failing hearts. The failing mouse heart switched towards $\alpha 2$ and $\beta 2$ while the failing human hearts increased the expression of $\alpha 1$ and $\beta 1$. Moreover, the transcriptional mechanism played a role in these changes in the mouse heart but unlikely so in the human heart. Our results did not allow us to address the potential role of the translational or posttranslational mechanisms, such as RNA-binding proteins, micro-RNAs or protein degradation pathways in the regulation of the AMPK protein levels in human failing hearts.

\section{Isoform specific activity of AMPK in the failing and non-failing heart}

We next determined whether the changes in the isoform expression affected the activity of AMPK containing the $\alpha 1$ or the $\alpha 2$ catalytic subunit in either mouse or human failing hearts. We observed that the phosphorylation of the $\mathrm{Thr}^{172}$ on the $\alpha$ subunits, a key determinant of AMPK activity, was increased tremendously in the failing hearts compared to sham or nonfailing hearts in both mice (2.3 fold) and humans (4 fold) (Figure 4A and 4E). The isoformspecific AMPK activity showed similar degree of activation for both isoforms (Figure 4, B- 
$\mathrm{C}, \mathrm{F}-\mathrm{G})$. Increases in isoform-specific AMPK activity could be attributed to the increased $\mathrm{Thr}^{172}$ phosphorylation and/or the increased expression of that particular isoform. To distinguish the two possibilities, we sought to determine the contribution of altered isoform protein amount by comparing the isoform-specific kinase activity of comparably phosphorylated complexes. To do so, we first immunoprecipitated the AMPK complex containing either the $\alpha 1$ or the $\alpha 2$ subunit with respective antibodies and then incubated the immunoprecipitate with the upstream kinase LKB1/STRADa/MO25a complex or $\lambda$ phosphatase to achieve maximal or minimal phosphorylation of the $\operatorname{Thr}^{172}$ on the $\alpha$-AMPK. As shown in Figure 4, the AMPK activity changed substantially in response to dephosphorylation and re-phosphorylation. In samples treated with LKB1 to achieve a comparable level of $\mathrm{Thr}^{172}$ phosphorylation the AMPK activity was greater in the failing human hearts (Figure 5) suggesting that a higher protein expression could increase the overall capacity of the kinase activity. The difference in AMPK activity in LKB1-treated mouse failing and sham hearts was less remarkable showing a modest increase of $\alpha 1$-AMPK activity but not total activity. The total amount of AMPK complexes in the hearts could not compared due to the inefficiency of immunoprecipitation of pan $\alpha$-AMPK antibody (Supp. Figure 2).

The relative amount of the $\alpha 1$ - versus $\alpha 2$-containing AMPK in each group of the hearts is difficult to assess due to the differences in the antibody affinity for the two isoforms. As the $\alpha 1$ - and $\alpha 2$-AMPK specific antibodies can completely pulled down the corresponding isoforms in both mice and human heart extracts we used the kinase activity after LKB1 treatment as a surrogate for the protein amount of each isoform (Figure 5A-B, Suppl. Figure 2 ). The relative contribution of $\alpha 1$-AMPK and $\alpha 2$-AMPK specific activity shown in Figure $5 \mathrm{C}$ was calculated from the data in Figure 4. In mouse hearts, the $\alpha 2$ complexes accounted for $\sim 2 / 3$ of total AMPK activity (sum of the $\alpha 1$ - and $\alpha 2$-AMPK activity) while the $\alpha 1$ complexes accounted for the remaining 30-35\%. This distribution did not change significantly in the failing mouse heart (Figure 5C). This is also consistent with the literature suggesting that $\alpha 2$ subunit accounts for the major portion of total AMPK activity in the rodent hearts [13] [15]. In the human hearts, however, the contribution of $\alpha 1$-AMPK activity were significantly higher (>40\%), $\alpha 1 / \alpha 2$-AMPK activity is $0.75 \pm 0.06$ in the non-failing hearts vs. $0.53 \pm 0.04$ in the mouse sham hearts $(\mathrm{P}<0.05)$, and it further increased to $50 \%$ $(1.11 \pm 0.11)$ in the failing human hearts $(\mathrm{P}<0.01$ vs. non-failing human hearts, $\mathrm{P}<0.01$ vs. mouse sham hearts, $0.63 \pm 0.14$ ). Thus, the human hearts have a greater amount of $\alpha 1$ AMPK activity compared to the rodent hearts.

\section{Discussion}

In this study we analyzed the AMPK isoform expression and activity in the heart during the development and in heart failure. We also compared the mouse heart to the human heart to determine whether the mouse heart is a good model for the human AMPK in the failing and non-failing hearts. There are several major findings of the study 1) AMPK expression is higher in the fetal heart than the adult, and part of the fetal expression profile of the AMPK isoforms reappears in the failing heart. 2) The protein expression and the activity of AMPK both increased significantly in the failing heart. 3) Changes in the specific isoforms are different in human failing heart compared to the mouse.

The AMPK cascade functions to maintain cellular energy homeostasis and regulate cell growth and survival [2] [19] [20]. Deletion of both isoforms of the catalytic subunit of AMPK is embryonic lethal suggesting a critical role of this pathway during the development. Here we found that the expression of AMPK was greater in the fetal heart, and in particular, the expressions of the $\alpha 1$ and $\gamma 2$ isoforms were 3 -fold higher in the E15 mouse hearts and the expression fell significantly in the neonatal hearts. It is interesting that the 
trough of expression for most of the isoforms is around the time of birth. However, the tissue collection process in the fetal and neonatal mice affected the $\mathrm{Thr}^{172}$ phosphorylation hence the AMPK activity we were unable to assess whether this pattern of expression was paralleled by the changes in the AMPK activity. Previous study by Makinde et al. observed an increase of AMPK activity in newborn rabbit hearts from day 1 to day 7 [21]. This may reflect an increase in AMPK phosphorylation during that period.

Since the $\alpha 1$ and $\gamma 2$ were minor isoforms for these subunits in the normal adult hearts, our results show a significant isoform switch for these subunits during the development. We also observed an upregulation of the $\gamma 2$ isoform expression in the failing heart suggesting a reversal of the $\gamma$ subunit isoforms toward the fetal pattern. The isoform-specific function of the $\gamma$ subunit in the heart is poorly understood. However, it has been shown that human mutations of $\gamma 2$-AMPK cause aberrant activation of AMPK activity resulting in glycogen storage, cardiac hypertrophy and arrhythmia. Thus, it is worth investigating whether the upregulation of the $\gamma 2$ isoform in the failing heart, observed both in mice and humans, is mechanistically involved in the development of pathological hypertrophy and heart failure.

Changes in the isoform-specific AMPK activity have been shown in rodent hearts during ischemia and pressure overload [7] [9] [22]. Here we find that the isoform distribution of AMPK in the human hearts is not identical to the mouse hearts and the changes in their expression in the human failing hearts only partially overlap with that of mouse failing hearts. Although the upregulation of $\gamma 2$ isoform were observed in both mouse and human failing hearts, the isoform expression for the $\alpha$ and $\beta$ subunits were changed in distinct directions. The mouse failing heart switched towards $\alpha 2$ and $\beta 2$ subunits while the human failing hearts expressed more $\alpha 1$ and $\beta 1$ subunits. We expect that these changes will not only increase the total amount of AMPK heterotrimers but also alter the subunit isoform composition of the kinase, i.e. more AMPK complexes in the failing mouse heart contain $\alpha 2$ and $\beta 2$ isoforms compared to the controls while in the failing human heart, AMPK heterotrimers containing $\alpha 1$ and $\beta 1$ isoforms are increased. By measuring the isoformspecific AMPK activity in samples after full phosphorylation of $\alpha$-AMPK, we found that in mouse heart $\alpha 2$ complexes accounted for the majority $(65 \%)$ of the AMPK activity with $\alpha 1$ complexes accounted for the remaining 35\%. In contrast, the human heart has higher $\alpha 1$ AMPK activity and it further increases in the failing heart where $\alpha 1$ - and $\alpha 2$-AMPK have equal contribution to the total activity. The functional significance of the altered composition of the $\alpha$ and $\beta$ subunit isoforms of the AMPK in the heart is unknown. However, it has been shown in non-cardiac cell types that AMPK $\alpha 2$-containing complexes are found in both the cytoplasm and the nucleus while AMPK $\alpha 1$-containing complexes are predominantly localized in the cytoplasm and have been also observed at the plasma membrane in some cell types such as carotid body cells [23] [24] [25]. Leptin treatment of $\mathrm{C} 2 \mathrm{C} 12$ cells caused the translocation of AMPK containing $\alpha 2$ and $\beta 2$ isoform into the nucleus after Thr172 phosphorylation of $\alpha 2$ whereas AMPK containing $\alpha 2$ and $\beta 1$ isoform was anchored in the cytoplasm through the myristoylation [26]. Therefore, future work delineating isoform specific functions of AMPK in the heart will be essential for better understanding of the signaling cascade in heart failure.

AMPK is activated in animal models of pathological hypertrophy and heart failure [7] [9] [27] although one study showed inactivation of AMPK by oxidative stress in the heart of spontaneous hypertensive rats [28]. In the present study we found a similar increase of AMPK activity in human failing hearts as that in mice and rats. Furthermore, our study suggests that increased phosphorylation of $\alpha$-AMPK is primarily responsible for the greater AMPK activity in the failing heart, which may be linked to changes in AMPK subunit composition rather than increased expression of AMPK. In mouse hearts, although the expression level of AMPK has changed in the failing heart, we detected only a modest 
increase of activity in the failing hearts when the tissue homogenates was treated with LKB1 to match the $\alpha$-AMPK phosphorylation in failing and non-failing samples. In the human failing hearts, however, both enhanced phosphorylation and increased AMPK protein expression contribute significantly to the upregulation of AMPK activity. Taken together, these observations raise the possibility that some of the regulatory mechanisms of the AMPK cascade in the heart are species specific. It is also worth noting that compared to the mouse study in which the tissue harvest process is well controlled the AMPK activities in human heart samples could be affected by the procedures applied to each individual patients. Nevertheless, the mouse and human results are directionally consistent. Since human heart samples are quite limited for research mouse heart will continue to be used for studies of AMPK in heart failure. Our study suggests that the results of rodent studies should be careful interpreted taking into consideration of possibly different regulatory mechanisms of AMPK cascade including isoform-specific function of AMPK across the species.

There are some other limitations of this study. Since we used cardiac tissue for all the experiments, we were unable to distinguish the changes of AMPK expression and activity originated from cardiac myocytes versus that from the non-myocyte, i.e. endothelial, fibroblasts or smooth muscle cells. By blotting for $\alpha$-sarcomeric actin, a protein expressed in cardiac myocyte but not in smooth muscle or non-muscle cells, we did confirm that the cardiac myocyte components in the protein extracts of failing and non-failing heart sample were comparable. Thus, our results are not due to the over representation of non-myocytes in the sample preparation. There are other limitations that should be taken into consideration, for example, we did not separate the LV from the RV in the mouse study, and the human study results might be influenced by the pharmacological treatments, the etiology of heart failure, and the age and gender of the patients. Due to the small number of patients included here we were unable to stratify for these factors, which warranted the future clinical study with a greater number of patients.

In summary, the protein level and the isoform distribution of AMPK in the heart change significantly during normal development as well as in heart failure. Our study has identified similarities and differences in the AMPK expression profile in response to chronic stresses during the development of heart failure in mice and humans. These results provide important basis for targeting AMPK for therapeutics, and furthermore, warrant investigation of isoform-specific function of AMPK in the heart.

\section{Supplementary Material}

Refer to Web version on PubMed Central for supplementary material.

\section{Acknowledgments}

We thank Dr. Federica del Monte for providing non-failing heart samples and Dr. Ming-Hui Zou for providing heart tissues of AMPK $\alpha 1$ and $\alpha 2$ null mice. This study was supported in part by grants from the National Institutes of Health: R01 HL067970 and HL088634.

\section{References}

1. Zhang BB, Zhou G, Li C. AMPK: an emerging drug target for diabetes and the metabolic syndrome. Cell Metab. 2009 May; 9(5):407-16. [PubMed: 19416711]

2. Hardie DG. AMPK and Raptor: matching cell growth to energy supply. Mol Cell. 2008 May 9; 30(3):263-5. [PubMed: 18471972]

3. Dasgupta B, Milbrandt J. AMP-activated protein kinase phosphorylates retinoblastoma protein to control mammalian brain development. Dev Cell. 2009 Feb; 16(2):256-70. [PubMed: 19217427] 
4. Carling D. The AMP-activated protein kinase cascade--a unifying system for energy control. Trends Biochem Sci. 2004 Jan; 29(1):18-24. [PubMed: 14729328]

5. Kemp BE, Stapleton D, Campbell DJ, Chen ZP, Murthy S, Walter M, et al. AMP-activated protein kinase, super metabolic regulator. Biochem Soc Trans. 2003 Feb; 31(Pt 1):162-8. [PubMed: 12546677]

6. Hue L, Rider MH. The AMP-activated protein kinase: more than an energy sensor. Essays Biochem. 2007; 43:121-37. [PubMed: 17705797]

7. Tian R, Musi N, D'Agostino J, Hirshman MF, Goodyear LJ. Increased adenosine monophosphateactivated protein kinase activity in rat hearts with pressure-overload hypertrophy. Circulation. 2001 Oct 2; 104(14):1664-9. [PubMed: 11581146]

8. Russell RR 3rd, Li J, Coven DL, Pypaert M, Zechner C, Palmeri M, et al. AMP-activated protein kinase mediates ischemic glucose uptake and prevents postischemic cardiac dysfunction, apoptosis, and injury. J Clin Invest. 2004 Aug; 114(4):495-503. [PubMed: 15314686]

9. Gundewar S, Calvert JW, Jha S, Toedt-Pingel I, Ji SY, Nunez D, et al. Activation of AMP-activated protein kinase by metformin improves left ventricular function and survival in heart failure. Circ Res. 2009 Feb 13; 104(3):403-11. [PubMed: 19096023]

10. Liao Y, Takashima S, Maeda N, Ouchi N, Komamura K, Shimomura I, et al. Exacerbation of heart failure in adiponectin-deficient mice due to impaired regulation of AMPK and glucose metabolism. Cardiovasc Res. 2005 Sep 1; 67(4):705-13. [PubMed: 15907819]

11. Zhang P, Hu X, Xu X, Fassett J, Zhu G, Viollet B, et al. AMP activated protein kinase-alpha2 deficiency exacerbates pressure-overload-induced left ventricular hypertrophy and dysfunction in mice. Hypertension. 2008 Nov; 52(5):918-24. [PubMed: 18838626]

12. Xing Y, Musi N, Fujii N, Zou L, Luptak I, Hirshman MF, et al. Glucose metabolism and energy homeostasis in mouse hearts overexpressing dominant negative alpha2 subunit of AMP-activated protein kinase. J Biol Chem. 2003 Aug 1; 278(31):28372-7. [PubMed: 12766162]

13. Stapleton D, Mitchelhill KI, Gao G, Widmer J, Michell BJ, Teh T, et al. Mammalian AMPactivated protein kinase subfamily. J Biol Chem. 1996 Jan 12; 271(2):611-4. [PubMed: 8557660]

14. Thornton C, Snowden MA, Carling D. Identification of a novel AMP-activated protein kinase beta subunit isoform that is highly expressed in skeletal muscle. J Biol Chem. 1998 May 15; 273(20): 12443-50. [PubMed: 9575201]

15. Cheung PC, Salt IP, Davies SP, Hardie DG, Carling D. Characterization of AMP-activated protein kinase gamma-subunit isoforms and their role in AMP binding. Biochem J. 2000 Mar 15; 346(Pt 3):659-69. [PubMed: 10698692]

16. Liao R, Jain M, Cui L, D'Agostino J, Aiello F, Luptak I, et al. Cardiac-specific overexpression of GLUT1 prevents the development of heart failure attributable to pressure overload in mice. Circulation. 2002 Oct 15; 106(16):2125-31. [PubMed: 12379584]

17. Karamanlidis G, Nascimben L, Couper GS, Shekar PS, del Monte F, Tian R. Defective DNA replication impairs mitochondrial biogenesis in human failing hearts. Circ Res. 2010 May 14; 106(9):1541-8. [PubMed: 20339121]

18. Lang T, Yu L, Tu Q, Jiang J, Chen Z, Xin Y, et al. Molecular cloning, genomic organization, and mapping of PRKAG2, a heart abundant gamma2 subunit of 5'-AMP-activated protein kinase, to human chromosome 7q36. Genomics. 2000 Dec 1; 70(2):258-63. [PubMed: 11112354]

19. Luo Z, Zang M, Guo W. AMPK as a metabolic tumor suppressor: control of metabolism and cell growth. Future Oncol. 2010 Mar; 6(3):457-70. [PubMed: 20222801]

20. Jager S, Handschin C, St-Pierre J, Spiegelman BM. AMP-activated protein kinase (AMPK) action in skeletal muscle via direct phosphorylation of PGC-1alpha. Proc Natl Acad Sci U S A. 2007 Jul 17; 104(29):12017-22. [PubMed: 17609368]

21. Makinde AO, Gamble J, Lopaschuk GD. Upregulation of 5'-AMP-activated protein kinase is responsible for the increase in myocardial fatty acid oxidation rates following birth in the newborn rabbit. Circ Res. 1997 Apr; 80(4):482-9. [PubMed: 9118478]

22. Li J, Coven DL, Miller EJ, Hu X, Young ME, Carling D, et al. Activation of AMPK alpha- and gamma-isoform complexes in the intact ischemic rat heart. Am J Physiol Heart Circ Physiol. 2006 Oct; 291(4):H1927-34. [PubMed: 16648175] 
23. Salt I, Celler JW, Hawley SA, Prescott A, Woods A, Carling D, et al. AMP-activated protein kinase: greater AMP dependence, and preferential nuclear localization, of complexes containing the alpha2 isoform. Biochem J. 1998 Aug 15; 334(Pt 1):177-87. [PubMed: 9693118]

24. Hallows KR, Kobinger GP, Wilson JM, Witters LA, Foskett JK. Physiological modulation of CFTR activity by AMP-activated protein kinase in polarized T84 cells. Am J Physiol Cell Physiol. 2003 May; 284(5):C1297-308. [PubMed: 12519745]

25. Evans AM, Mustard KJ, Wyatt CN, Peers C, Dipp M, Kumar P, et al. Does AMP-activated protein kinase couple inhibition of mitochondrial oxidative phosphorylation by hypoxia to calcium signaling in O2-sensing cells? J Biol Chem. 2005 Dec 16; 280(50):41504-11. [PubMed: 16199527]

26. Suzuki A, Okamoto S, Lee S, Saito K, Shiuchi T, Minokoshi Y. Leptin stimulates fatty acid oxidation and peroxisome proliferator-activated receptor alpha gene expression in mouse $\mathrm{C} 2 \mathrm{C} 12$ myoblasts by changing the subcellular localization of the alpha2 form of AMP-activated protein kinase. Mol Cell Biol. 2007 Jun; 27(12):4317-27. [PubMed: 17420279]

27. Li HL, Yin R, Chen D, Liu D, Wang D, Yang Q, et al. Long-term activation of adenosine monophosphate-activated protein kinase attenuates pressure-overload-induced cardiac hypertrophy. J Cell Biochem. 2007 Apr 1; 100(5):1086-99. [PubMed: 17266062]

28. Dolinsky VW, Chan AY, Robillard Frayne I, Light PE, Des Rosiers C, Dyck JR. Resveratrol prevents the prohypertrophic effects of oxidative stress on LKB1. Circulation. 2009 Mar 31; 119(12):1643-52. [PubMed: 19289642] 


\section{Highlights}

1. AMPK expression is higher in the fetal heart than the adult.

2. Part of the fetal expression profile of the AMPK isoforms reappears in the failing heart.

3. The protein expression and the activity of AMPK both increased significantly in the failing heart.

4. Changes in the specific isoforms are different in human failing heart compared to the mouse. 


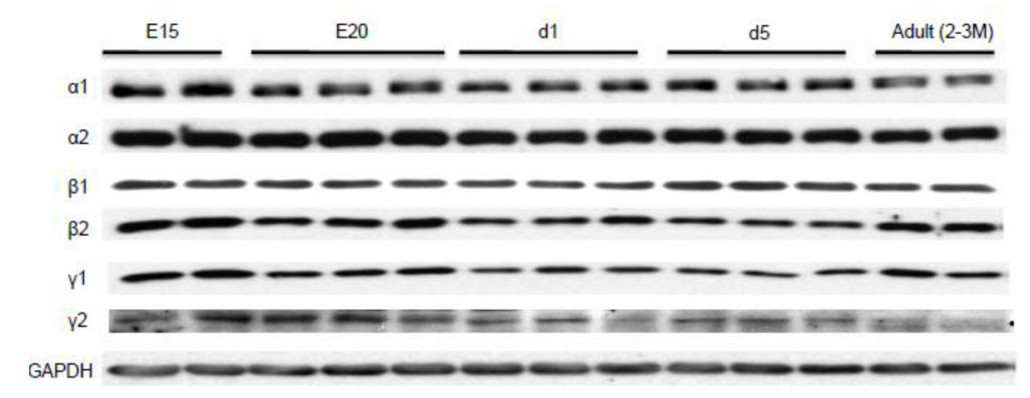

B
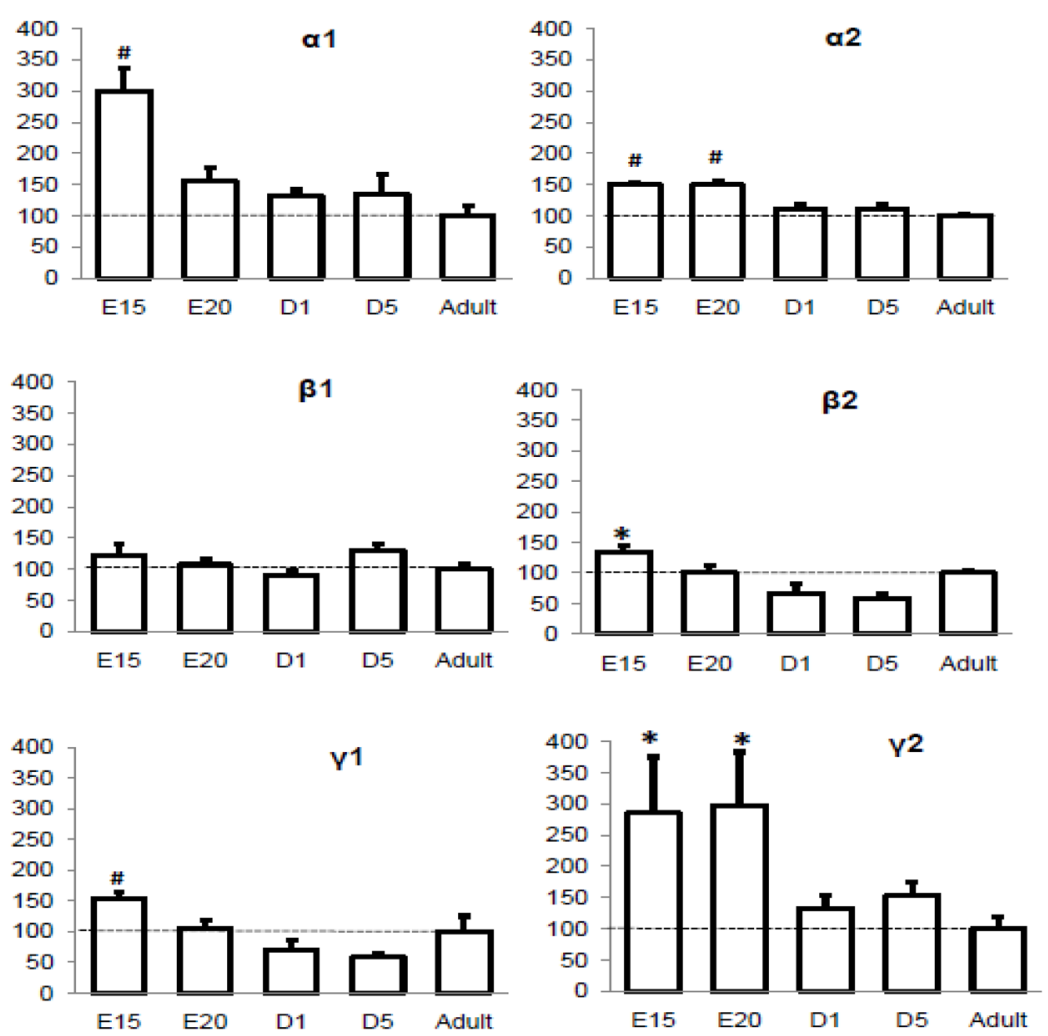

Figure 1. Protein expression profile of AMPK subunit isoform in the developing and adult mouse hearts

A. Representative Western Blot. B. Quantitation of AMPK subunit isoforms. The Western blots were probed by antibodies against AMPK subunit isoforms. The expression was normalized to GAPDH and data were expressed as \% adult level. E15, Fetal 15 day; E20, Fetal 20 day; d1, Neonate 1 day; d5, Neonate 5 day. ${ }^{*} \mathrm{P}<0.05$ versus adult; ${ }^{\#} \mathrm{P}<0.01$ versus adult. The mean values \pm SD are shown. 
A

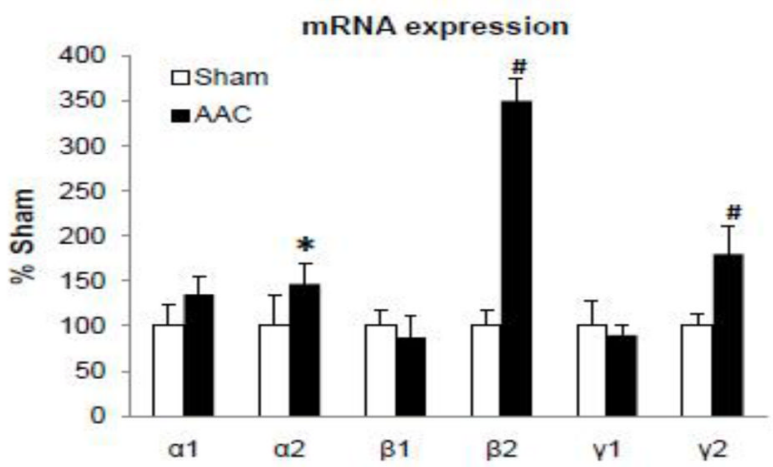

B
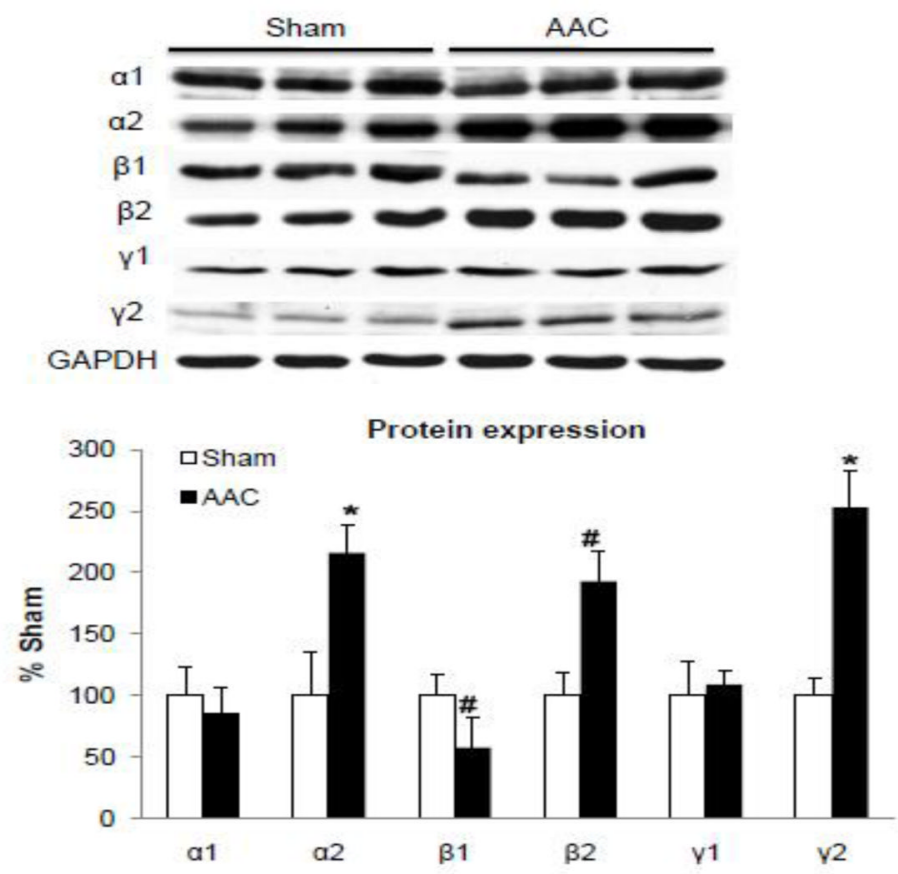

Figure 2. Expression profile of AMPK subunit isoform mRNA (A) and protein (B) levels in failing mouse hearts

A. mRNA levels were assessed by real time PCR and normalized to $18 \mathrm{~S}$ rRNA. Data were expressed as \% sham. B. Protein levels were assessed by Western blots and normalized to GAPDH and data were expressed as $\%$ sham. $* \mathrm{P}<0.05$ versus sham; ${ }^{\#} \mathrm{P}<0.01$ versus sham. The mean values \pm SD are shown. 
A mRNA expression

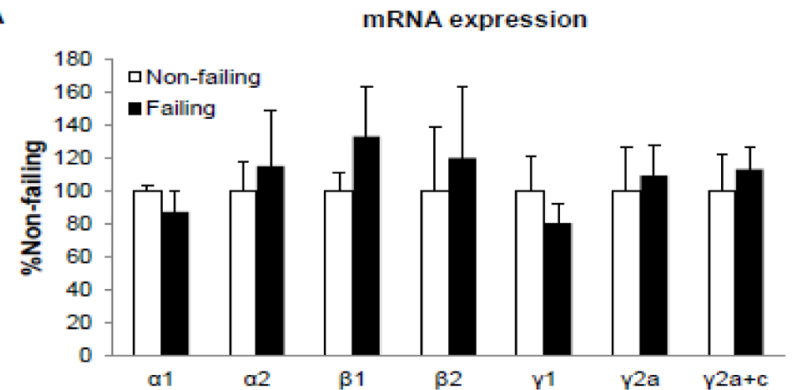

B

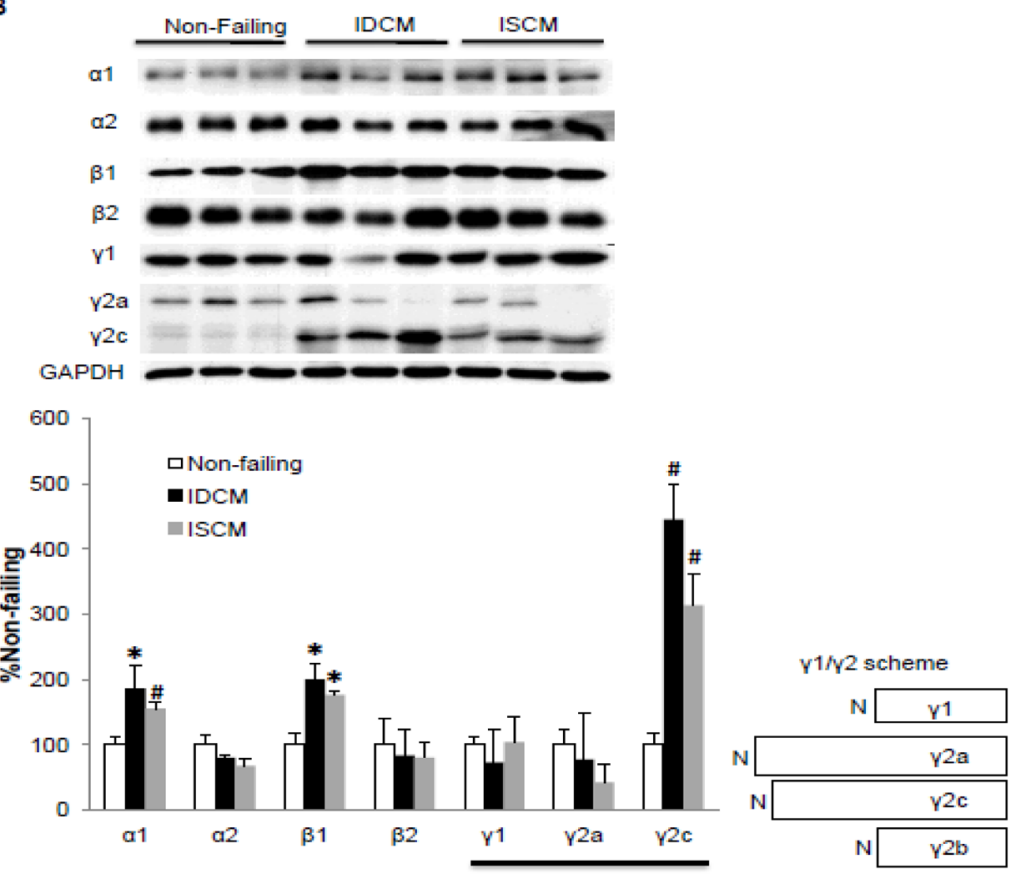

Figure 3. Expression profile of AMPK subunit isoform mRNA (A) and protein (B) levels in failing human hearts

A. mRNA levels were assessed by real time PCR and normalized to $18 \mathrm{~S}$ rRNA. Data were expressed as \% non-failing. B. Protein levels were assessed by Western blots. The right panel shows a schematic diagram of three splice variants of AMPK $\gamma 2$ subunit in the human heart. The expression was normalized to GAPDH and data were expressed as \% non-failing. ${ }^{*} \mathrm{P}<0.05$ versus non-failing; ${ }^{*} \mathrm{P}<0.01$ versus non-failing. The mean values $\pm \mathrm{SD}$ are shown. IDCM, Idiopathic Dilated Cardiomyopathy; ISCM: Ischemic Cardiomyopathy 

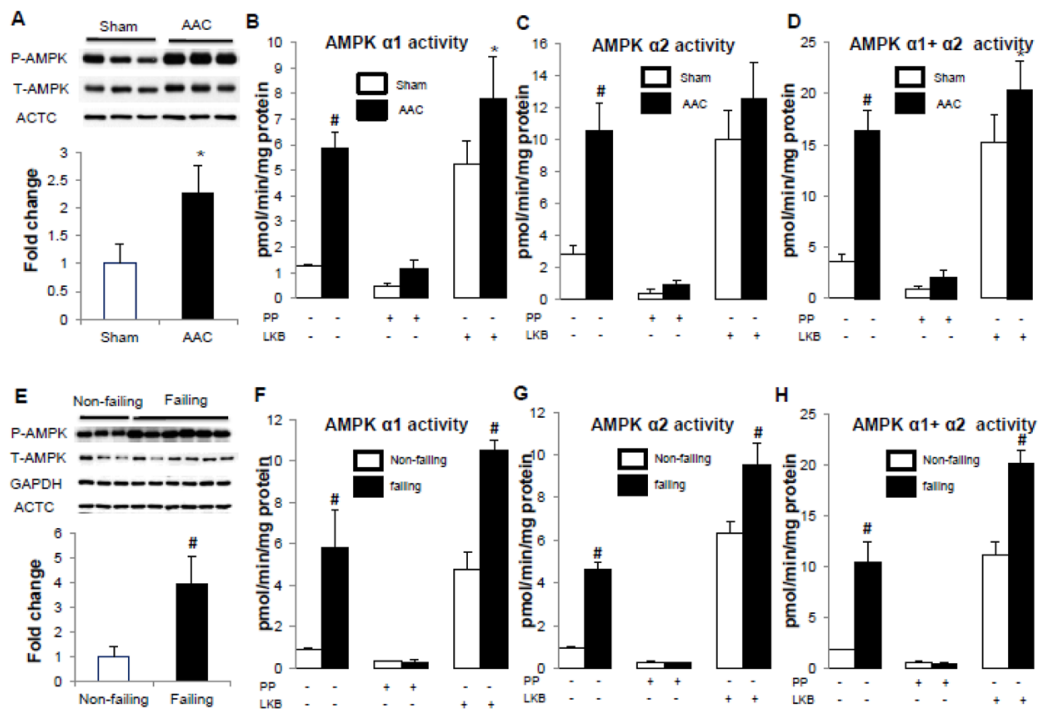

Figure 4. AMPK activity in the failing hearts of mice (A-D) and humans (E-H)

A and E. Phophorylation of T172 of AMPK $\alpha$ subunits normalized against its corresponding total protein. GAPDH or ACTC ( $\alpha$-sarcomeric actin) was used as a loading control. Data were expressed as fold change of the sham or non-failing group. B and F. $\alpha 1$-AMPK specific activity with or without de-phosphorylation by lambda phophatase (PP) followed by re-phosphorylation by LKB1/STRAD $\alpha /$ MO25 $\alpha$ complex (LKB). C and G. $\alpha 2-A M P K$ specific activity determined by the same procedure as described above. D and $\mathrm{H}$. Total AMPK activity was obtained by sum of AMPK $\alpha 1$ and $\alpha 2$ specific activity. $* \mathrm{P}<0.05$ versus sham or non-failing; ${ }^{\#} \mathrm{P}<0.01$ versus sham or non-failing. The mean values $\pm \mathrm{SD}$ are shown. + , treatment with PP or LKB; - , no treatment. 
A

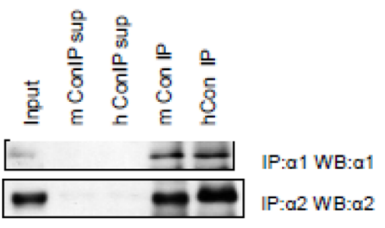

c

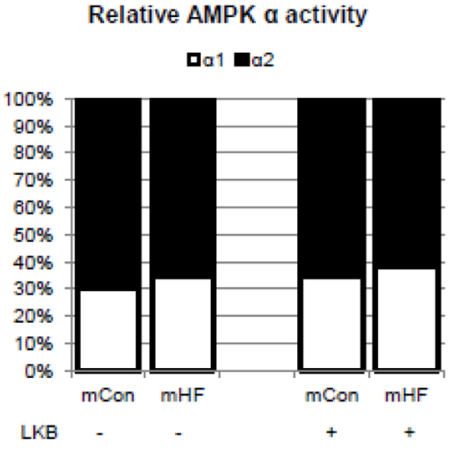

B

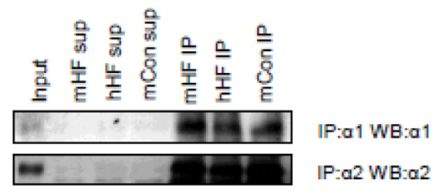

Relative AMPK $\alpha$ activity $\mathbf{Q}_{\alpha 1} \mathbf{W}_{\alpha 2}$

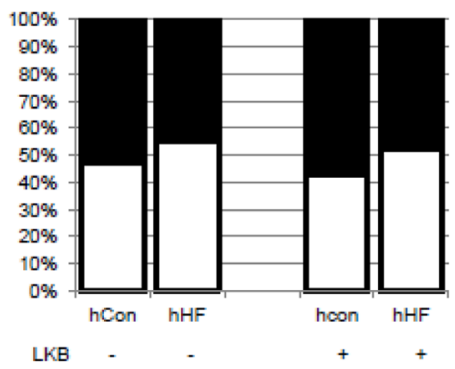

Figure 5. Relative contribution of AMPK $\alpha 1$ and $\alpha 2$ specific activity in the failing heart AMPK complexes containing $\alpha 1$ or $\alpha 2$ in the extracts can be completely immunoprecipitated by antibodies specific to AMPK $\alpha 1$ or $\alpha 2$ isoform in control (A) and failing (B) hearts of mice and humans. C. The immunoprecipiates were processed for AMPK activity measurement with or without phosphorylation by LKB1/STRAD $\alpha / \mathrm{MO} 25 \alpha$ complex. The relative contribution of $\alpha 1$ - vs $\alpha 2$-AMPK was re-calculated from Figure 4B-D and $\mathrm{F}-\mathrm{H}$. The AMPK $\alpha 1$ - or $\alpha 2$ - specific activity was normalized by AMPK $\alpha 1+2$ activity. Data were expressed as \%100. Sup, supernatant; mCon, mouse sham hearts; hCon, human non-failing hearts; mHF, mouse failing hearts; hHF, human failing hearts 
Table 1

characteristics of the mouse failing hearts

\begin{tabular}{|l|c|c|}
\hline & Sham & AAC \\
\hline Animal number & 5 & 5 \\
\hline Body Weight, g & $26 \pm 1.5$ & $26 \pm 2.6$ \\
\hline Heart Weight, mg & $108 \pm 4.6$ & $207 \pm 20.2$ \\
\hline HW/BW, mg/g & $4.1 \pm 0.21$ & $8.0^{\dagger} \pm 1.26$ \\
\hline Heart Rate, beat/min & $671 \pm 31.3$ & $600 \pm 40.1$ \\
\hline LVAWD, mm & $0.92 \pm 0.078$ & $1.29^{\dagger} \pm 0.078$ \\
\hline LVIDD, mm & $2.69 \pm 0.208$ & $3.44^{\dagger} \pm 0.292$ \\
\hline LVIDS, mm & $0.98 \pm 0.200$ & $2.53^{\dagger} \pm 0.136$ \\
\hline Ejection Fraction, $\%$ & $92.5 \pm 2.82$ & $51.3^{\dagger} \pm 4.66$ \\
\hline Fractional Shortening, $\%$ & $63.9 \pm 4.80$ & $25.6^{\dagger} \pm 3.01$ \\
\hline
\end{tabular}

HW/BW: Heart weight are normalized to body weight

LVAWD: End-diastolic anterior wall thickness of the left ventricle

LVIDS: End-systolic internal diameter of the left ventricle

Date are shown as mean $\pm \mathrm{SD}$.

${ }^{\dagger} \mathrm{P}<0.01$ versus sham. 
Table 2

characteristics of the group study

\begin{tabular}{|c|c|c|}
\hline & Non-failing & Failing \\
\hline Number of group & 8 & 9 \\
\hline Gender & $3 \mathrm{M} / 5 \mathrm{~F}$ & $7 \mathrm{M} / 2 \mathrm{~F}$ \\
\hline Age in years Median (Min-Max) & $59(42-71)$ & $59(22-67)$ \\
\hline Number of IDCM & N/A & 6 \\
\hline Number of ISCM & N/A & 3 \\
\hline Ejection Fraction, \% & N/A & $16 \pm 2.2$ \\
\hline
\end{tabular}

IDCM: Idiopathic Dilated Cardiomyopathy

ISCM: Ischemic Cardiomyopathy 
